\title{
Standounkt
}

Industrie 4.0

\section{Eine selbsterfüllende Prophezeiung?}

\author{
Fortschritt durch Digitalisierung - so das Erfolgsversprechen \\ der Industrie 4.0. Doch ist die Digitalisierung auch mit zahlreichen \\ gesellschaftlichen Risiken verknüpft. Damit sich die Versprechen \\ erfüllen, sind sozial-ökologische Visionen und ein breiter politischer \\ Diskurs notwendig. \\ Von Vivian Frick
}

B ald schon sind wir alle mit selbstfahrenden Autos unterwegs. Beleuchtungssysteme, Heizungen und Waschmaschinen besprechen unseren Alltag, während wir dank 24-Stunden-Lieferservice per Drohne bequem vom Sofa aus einkaufen. Arbeit und Produktion übernehmen währenddessen Maschinen und Algorithmen.

Solche Zukunftsbilder zeichnet der Diskurs um Digitalisierung und ,Industrie 4.0'. Letzterer wird primär durch große Wirtschaftsverbände, Unternehmen und politische Akteure gestaltet (Pilgrim et al. 2017). Der Fokus liegt auf Wirtschaftlichkeit, während soziale und Umweltaspekte eher stiefmütterlich behandelt werden: Industrie 4.0 soll Profite in Milliardenhöhe generieren, bei Nichtumsetzung drohen ebenso hohe Verluste (Roland Berger 2016). Das Rennen hat unausweichlich begonnen und wer nicht auf den Zug aufspringt, bleibt auf der Strecke.

Akzeptieren wir diese Prognosen bereits vor ihrem Eintreffen als Realität, so können sie schnell zur selbsterfüllenden Prophezeiung werden. Doch welche dieser Veränderungen erachten wir als Gesellschaft eigentlich für sinnvoll und erstrebenswert? Die Industrie 4.0 verspricht Effizienzsteigerung, dadurch günstigere Produktion von Gütern, Anpassung an neue Konsumbedürfnisse und eine Expansion der Wirtschaftsleistung (Plattform Industrie 4.0 2017). Sind dies tatsächlich gesellschaftliche Verbesserungen oder holen wir uns damit das trojanische Pferd in den virtuellen Hof?
Ich möchte hier auf zwei der gesellschaftlichen Risiken eingehen. Einerseits ist die Debatte um den Datenschutz von einer Lösung weit entfernt. Wer sich im Netz bewegt, hinterlässt bei jeder Handlung Spuren, während sich auch im Alltag die Beobachtung u. a. durch Kameras ausweitet. Die so entstehenden Daten gefährden Persönlichkeitsrechte und ermöglichen eine Überwachung ungekannten Ausmaßes.

Andererseits gilt es, ökologische Auswirkungen der Industrie $4.0 \mathrm{zu}$ bedenken. Diese verspricht zunächst die „Dematerialisierung “, unter anderem durch "Clouds“. Diese Begriffe suggerieren eine Leichtigkeit, welche mit dem Ressourcenhunger digitaler Technologien wenig gemein hat. Durch Industrie 4.0 wird ein immenser Anstieg des Rohstoffbedarfes erwartet (BDI 2017; Pilgrim et al. 2017). Wir verlagern unseren Überkonsum im besten Fall von Kohle und Erdöl zu seltenen Erden, Lithium oder Kupfer (siehe den Beitrag von Michael Reckordt im Heft S. 21). Zusätzlich wird durch Industrie 4.0 die Produktion von Gütern ausgeweitet, was Konsumdruck erzeugt, während Online-Shopping ein extrem zeit- und kostensparendes Konsumieren ermöglicht.

Was diese beiden Probleme gemeinsam haben, ist ihre mangelnde Sichtund Fassbarkeit. Es fällt schwer, sich vorzustellen, wieviel Material und Energie für die Produktion allein eines Smartphones aufgewendet werden, oder wer unsere Daten wo, wie und wozu speichert. Die psychologische Distanz, ein
Begriff aus der Klimaforschung, erklärt, warum wir den Versprechen der Digitalisierung so bereitwillig glauben: Der Energieverbrauch findet weit entfernt in Rechenzentren statt. Die sozialen Auswirkungen sind vor allem in Minen und auf Müllhalden des globalen Südens zu spüren. Hackerangriffe, Überwachungsstaat oder Eingriffe in die Privatsphäre erscheinen uns in ferner Zukunft oder werden persönlich als unwahrscheinlich wahrgenommen. Das Bewusstsein für die materielle "Schwere“ und gesellschaftliche Gefahren der Digitalisierung muss daher aktiv gefördert werden, um diese umweltfreundlich und sozialverträglich gestalten zu können.

Aktuell wird Industrie 4.0 überwiegend zur Vertretung wirtschaftlicher Interessen vorangetrieben. Das Potenzial, sie stattdessen maßvoll für eine sozialökologische Gesellschaft einzusetzen, wäre vorhanden, wie dieses Heft zeigt. Zugleich stellt sich die Frage, ob der digitale „Fortschritt“ wirklich immer einer ist - oder ob man sich diesem auch mal verwehren darf. Eine verstärkte Inklusion gesellschaftlicher und ökologischer Akteure in die Debatte rund um Industrie 4.0 ist überfällig. Damit sich am Ende die „richtigen“ Prophezeiungen erfüllen, brauchen wir eine größere Vielfalt an Visionen - und einen politischen Diskurs darüber, welche davon Normalität werden sollen und welche nicht.

\section{Literatur}

BDI (2017): Digitalisierung erhöht Handlungsbedarf bei der Rohstoffsicherung. http://bdi.eu/

Pilgrim, H./Groneweg, M./Reckordt, M. (2017): Ressourcenfluch 4.0: Die sozialen und ökologischen Auswirkungen von Industrie 4.0 auf den Rohstoffsektor. Berlin.

Plattform Industrie 4.0 (2017): Was ist Industrie 4.0? www. plattform-i40.de

Roland Berger (2016): The Industrie 4.0 transition quantified. www.rolandberger.com

\section{AUTORIN + KONTAKT}

Vivian Frick ist wissenschaftliche Mitarbeiterin am Zentrum für Technik und Gesellschaft der TU Berlin.

Zentrum für Technik und Gesellschaft (ZTG), Hardenbergstr. 16-18, 10623 Berlin. Tel.: +493031429812, E-Mail: frick@ztg.tu-berlin.de and redistributing the material in any medium or format, provided the original work is properly cited, it is not used for commercial purposes and it is not remixed, transformed or built upon. The access to the digital version of this article is reserved to subscribers of ÖkologischesWirtschaften until two years after the date of publication; after two years it is available to all readers. 